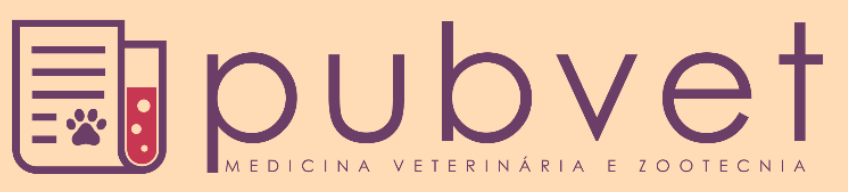

https://doi.org/10.31533/pubvet.v15n03a771.1-8

\title{
Similaridade florística de remanescentes de Cerrado da região Norte, Amazônia Legal
}

\author{
Bruno Aurélio Campos Aguiar ${ }^{*} *$, Marcos Vinicius Cardoso Silva $^{2}{ }^{\circ}$, Bárbara Gomes Ferreira ${ }^{3}{ }^{\circ}$, \\ Maurilio Antônio Varavallo ${ }^{\circ}{ }^{\circ}$, Thamires Marques Moura ${ }^{\circ}{ }^{\circ}$, André Ferreira dos $\operatorname{Santos}^{60}$, \\ Yasmim de Andrade $\operatorname{Ramos}^{7}{ }^{\circ}$, Priscila Bezerra de $\operatorname{Souza}^{8}{ }^{\circ}$

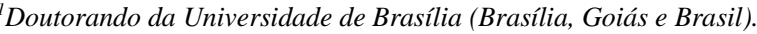 \\ ${ }^{2}$ Mestrando da Universidade Federal do Tocantins (Gurupi, Tocantins e Brasil). \\ ${ }^{3}$ Engenheira Civil, Mestre em Ciências Florestais e Ambientais (UFT). \\ ${ }^{4}$ Professor da Universidade Federal do Tocantins (Colegiado de Engenharia Florestal, Laboratório de Microbiologia), Gurupi, Tocantins, Brasil. \\ ${ }^{5}$ Doutoranda do Instituto Federal Goiano, Campus Rio Verde (Rio Verde, Goiás e Brasil) - \\ ${ }^{6}$ Professor da Universidade Federal do Tocantins (Colegiado de Engenharia Florestal), Gurupi, Tocantins, Brasil \\ ${ }^{7}$ Engenheira Florestal e Mestre em Ciências Florestais pela Universidade Federal do Tocantins - \\ ${ }^{8}$ Professora da Universidade Federal do Tocantins (Colegiado de Engenharia Florestal, Laboratório de Sementes), Gurupi, Tocantins, Brasil \\ *Autor para correspondência, E-mail: aguiar.florestal@gmail.com
}

Resumo. Considerando a abundância de espécies do domínio Cerrado e principalmente da fitofisionomia cerrado sensu stricto, torna-se importante estudos para analisar e compreender melhor essa riqueza. Portanto, visando conhecer e entender melhor essa riqueza e diversidade, o estudo foi realizado em cinco áreas de cerrado sensu stricto pertencentes a três regiões do norte da Amazônia Legal. Objetivou-se analisar a composição florística das espécies arbustivas-arbóreas de remanescentes de cerrado sensu stricto localizados em diferentes regiões e municípios do Tocantins, além de comparar a similaridade entre as áreas. Foram instaladas quarenta e três parcelas amostrais de 10x100 m, totalizando 4,3 hectares. Área 1 - oito parcelas de 10x100 m, perfazendo 0,8 ha, região centro-oeste, em Monte do Carmo. Área 2 - oito parcelas de 10x100 m, perfazendo 0,8 ha, região centro-oeste, em Santa Rita do Tocantins. Área 3 - dez parcelas de 10x100 m, perfazendo 1,0 ha, região central, em Divinópolis do Tocantins. Área 4 - dez parcelas de 10x100 m, perfazendo 1,0 ha, região Parque Estadual do Jalapão, em Santa Rita do Tocantins e Área 5 - sete parcelas de 10x100 m, perfazendo 0,7 ha, região Parque Estadual do Jalapão, em Mateiros. Foram amostrados todos os indivíduos com circunferência a altura do peito $\geq$ a $20 \mathrm{~cm}$, mensurado a $1,30 \mathrm{~m}$ do solo. Foram encontrados nas cinco áreas de cerrado sensu stricto, um total de 7.069 indivíduos, 264 espécies, pertencentes a 110 gêneros, incluídas em 46 famílias. A matriz para a análise de similaridade florística englobou 264 espécies registradas nas cinco áreas de cerrado sensu stricto, sendo área 1 (A1) 88 espécies, área 2 (A2) 53 espécies, área 3 (A3) 45 espécies, área 4 (A4) 62 espécies e área 5 (A5) 16 espécies. A heterogeneidade observada entre as áreas avaliadas indica que as espécies desta fisionomia de cerrado se caracterizam pela distribuição espacial em "mosaicos", com um limite máximo de 100 espécies por área estudada, e mesmo as populações existentes em áreas próximas apresentam-se floristicamente e estruturalmente diferenciadas.

Palavras-chave: Cerrado, composição florística, riqueza

\section{Floristic similarity of remanescents from Cerrado in from north regions of the legal Amazon}

Abstract. Considering the abundance of species from the Cerrado domain and especially from the cerrado sensu stricto phytophysiognomy, studies to analyze and better understand this richness become important. Therefore, in order to better understand and understand this 
richness and diversity, the study was carried out in five areas of cerrado sensu stricto belonging to three regions in the north of the Legal Amazon. The objective was to analyze the floristic composition of shrub-tree species of remnants of cerrado sensu stricto located in different regions and municipalities of Tocantins, in addition to comparing the similarity between the areas. Forty-three sampling plots of 10x100 m were installed, totaling 4.3 hectares. Area 1 eight plots of 10x100 m, totaling 0.8 ha, in the central-west region, in Monte do Carmo. Area 2 - eight plots of 10x100 m, totaling 0.8 ha, in the central-west region, in Santa Rita do Tocantins. Area 3 - ten plots of $10 \times 100 \mathrm{~m}$, totaling 1.0 ha, in the central region, in Divinópolis do Tocantins. Area 4 - ten plots of 10x100 m, totaling 1.0 ha, in the Jalapão State Park region, in Santa Rita do Tocantins and Area 5 - seven plots of 10x100 m, totaling 0.7 ha, in the Jalapão State Park region, in Mateiros. All individuals were sampled with circumference at breast height $\geq 20 \mathrm{~cm}$, measured at $1.30 \mathrm{~m}$ from the ground. In the five areas of cerrado sensu stricto, a total of 7,069 individuals, 264 species, belonging to 110 genera, included in 46 families, were found. The matrix for the analysis of floristic similarity encompassed 264 species recorded in the five areas of cerrado sensu stricto, being area 1 (A1) 88 species, area 2 (A2) 53 species, area 3 (A3) 45 species, area 4 (A4) 62 species and area 5 (A5) 16 species. The heterogeneity observed between the areas evaluated indicates that the species of this cerrado physiognomy are characterized by the spatial distribution in "mosaics", with a maximum limit of 100 species per studied area, and even the existing populations in nearby areas are floristically and structurally differentiated.

Keywords: Cerrado, floristic composition, wealth

\section{Introdução}

O Cerrado é o segundo maior bioma em área do continente sul americano, ocupa $22 \%$ do território brasileiro e possui alto nível de endemismo, abrigando 11.627 espécies de plantas nativas catalogadas (BRASIL, 2019). Sua localização geográfica territorial no Brasil estabelece fronteira com os demais biomas, favorecendo a inter-relação das espécies presentes com espécies de outras localidades, contribuindo nos valores de riqueza, diversidade e endemismo (Ferreira et al., 2015).

A peculiaridade da vegetação do Cerrado e sua diversidade de fisionomias podem ser explicadas por variações das características dos solos, pela topografia, estação seca bem definida, regime de fogo e por interferências humanas (Ratter et al., 2003). Considerando a abundância de espécies na fitofisionomia Cerrado sensu stricto e sabendo que a antropização impacta diretamente a diversidade de áreas nativas, tornam-se importantes estudos para analisar e compreender melhor sua riqueza.

A biodiversidade é a combinação de alterações bióticas desde o nível de genes a ecossistema (Purvis \& Hector, 2000), que se organizam em comunidades e são constituídas por dois importantes componentes: diversidade alfa e beta. A diversidade alfa é expressa pelo número de indivíduos e a abundância de espécies em uma área restrita com um habitat levemente uniforme. Já a diversidade beta está relacionada com alterações na composição de espécies e sua riqueza entre áreas de uma comunidade (Magurran, 1998).

Como o Tocantins é um dos estados brasileiros com maior área coberta pelo Cerrado, além de ser considerado o que apresenta os maiores índices de vegetação remanescente, com 79\% (Sano et al., 2010), objetivou-se analisar a composição florística das espécies arbustivas-arbóreas de remanescentes de Cerrado sensu stricto localizados em diferentes regiões tocantinenses, além de comparar a similaridade entre as áreas, visando dar subsídio para estratégias de manejo e conservação de sua vegetação lenhosa.

\section{Material e métodos}

O estudo foi realizado em cinco áreas de cerrado sensu stricto, no período de junho de 2015 a janeiro de 2016, em quatro municípios (Divinópolis do Tocantins, Mateiros, Monte do Carmo e Santa Rita do Tocantins), localizados em diferentes regiões do estado do Tocantins (SEPLAN, 2015), conforme descrito na Tabela 1.

Os dados amostrados foram obtidos pela realização de inventário florestal nestas cinco áreas de remanescentes de cerrado sensu stricto apresentadas, onde a vegetação do componente arbustivoarbóreo foi avaliada quantitativamente pelo método de parcelas (Mueller-Dombois \& Ellenberg, 1974). 
$\mathrm{Na}$ área um foram instaladas oito parcelas de $10 \times 100 \mathrm{~m}$, perfazendo 0,8 ha, na área dois foram oito parcelas de $10 \times 100 \mathrm{~m}$, perfazendo 0,8 ha, na área três foram dez parcelas de $10 \times 100 \mathrm{~m}$, perfazendo 1,0 ha, na área quatro foram dez parcelas de $10 \times 100 \mathrm{~m}$, perfazendo 1,0 ha e na área cinco foram sete parcelas de $10 \times 100 \mathrm{~m}$, perfazendo 0,7 ha, totalizando 43 parcelas amostrais e 4,3 hectares amostrados.

Tabela 1. Municípios, regiões, tipo de solo, clima, coordenadas geográficas e área e das áreas de estudo no estado do Tocantins.

\begin{tabular}{|c|c|c|c|c|c|}
\hline Áreas de estudo & Município & Região e tipo de solo & $\begin{array}{c}\text { Clima }^{1} \text { e médias } \\
\text { de temperatura } \\
\text { anual }\end{array}$ & $\begin{array}{l}\text { Médias de altitude e } \\
\text { precipitação pluviométrica }\end{array}$ & $\begin{array}{l}\text { Coordenadas } \\
\text { Geográficas }\end{array}$ \\
\hline $01-$ & $\mathrm{Mc}$ & $\begin{array}{l}\text { Centro-oeste / latossolo } \\
\text { vermelho-escuro }\end{array}$ & $\begin{array}{l}\text { C2wA'a', } \\
26 \text { a } 28^{\circ} \mathrm{C}\end{array}$ & $295 \mathrm{~m} / 1700$ a $1800 \mathrm{~mm}$ & $\begin{array}{r}10^{\circ} 46^{\prime} \\
48^{\circ} 3\end{array}$ \\
\hline $\begin{array}{l}02 \text { - Fazenda Nossa } \\
\text { Senhora Consoladora }\end{array}$ & $\begin{array}{l}\text { Santa Rita do } \\
\text { Tocantins }\end{array}$ & $\begin{array}{l}\text { Centro-oeste/ } \\
\text { plintossolo }\end{array}$ & $\begin{array}{l}\text { C2wA'a" } \\
26 \text { a } 28^{\circ} \mathrm{C}\end{array}$ & $295 \mathrm{~m} / 1700$ a $1800 \mathrm{~mm}$ & $\begin{array}{l}10^{\circ} 28^{\prime} 19,94 " \mathrm{~S} \text { e } \\
47^{\circ} 57^{\prime} 37,97^{\prime \prime} \mathrm{W}\end{array}$ \\
\hline $\begin{array}{l}03 \text { - Fazenda } \\
\text { Remanso }\end{array}$ & $\begin{array}{l}\text { Divinópolis do } \\
\text { Tocantins }\end{array}$ & Central / plintossolo & $\begin{array}{l}\mathrm{C} 2 \mathrm{w} \\
26 \mathrm{a}\end{array}$ & $270 \mathrm{~m} / 2100 \mathrm{~mm}$ & $\begin{array}{l}9^{\circ} 35^{\prime} 5 \\
49^{\circ} 29^{\prime}\end{array}$ \\
\hline $\begin{array}{l}04 \text { - Fazenda Santa } \\
\text { Bárbara }\end{array}$ & $\begin{array}{l}\text { Santa Rita do } \\
\text { Tocantins }\end{array}$ & $\begin{array}{l}\text { Parque Estadual do Jalapão/ } \\
\text { neossolo quartzarênico }\end{array}$ & $\begin{array}{l}\text { C2wA'a” } \\
26 \text { a } 28^{\circ} \mathrm{C}\end{array}$ & 355 m / 1900 a 2000 mm & $\begin{array}{l}10^{\circ} 47,58,01 \text { ' S e } \\
49^{\circ} 21,49,59 \text { " W }\end{array}$ \\
\hline 05 - Fazenda Lote 19 & Mateiros & $\begin{array}{l}\text { Parque Estadual do } \\
\text { Jalapão / plintossolo }\end{array}$ & $\begin{array}{l}\text { C2w2A'a” } \\
26 \text { a } 28^{\circ} \mathrm{C}\end{array}$ & $493 \mathrm{~m} / 1400$ a $1500 \mathrm{~mm}$ & $\begin{array}{l}11^{\circ} 12^{\prime}, 5,51^{\prime \prime} \mathrm{S} \mathrm{e} \\
46^{\circ} 21^{\prime} 49,59^{\prime \prime} \mathrm{W}\end{array}$ \\
\hline
\end{tabular}

${ }^{1}$ Baseado na classificação de Thornthwaite.

Após distribuição das parcelas, todos os indivíduos com circunferência a altura do peito (CAP) a $1,30 \mathrm{~m}$ do solo igual ou superior a $20 \mathrm{~cm}$ foram amostrados. A identificação das espécies arbustivasarbóreas, sempre que possível foi realizada em campo pela coleta de material botânico, se não identificados na área de coleta, comparava-se o material botânico coletado com o material do Herbário HTO, campus de Porto Nacional, literatura especializada e consulta a especialista, quando necessário. O sistema Angiosperm Phylogeny Group IV foi utilizado para classificar as famílias botânicas (APG IV, 2016). Os nomes das espécies e seus respectivos autores e sinonímias foram confirmados e atualizados pelo site da Lista de Espécies da Flora do Brasil (REFLORA, 2020).

A composição florística do presente estudo foi comparada entre as cinco áreas inventariadas. Para o cálculo da similaridade foi utilizado o índice de Sorensen (Ss), que compara qualitativamente a presença e ausência de espécies na área. Este índice varia em uma escala de 0 a 1 , sendo que a similaridade é considerada elevada se os valores superarem 0,5 (Kent \& Coker, 1992; Magurran, 1998). Para análise dos dados foi utilizado o Programa FITOPAC 2.1.2 (Shepherd, 2010), enquanto a interpretação foi feita por meio do método média de grupo (UPGMA) (Sneath \& Sokal, 1966). A criação dos dendogramas foi realizada com o programa MVSP3.13 (Kovach, 2007) e os gráficos foram gerados através do MSExcel.

\section{Resultados e discussão}

Foram amostrados nas cinco áreas de cerrado sensu stricto um total de 7.069 indivíduos, 264 espécies, pertencentes a 110 gêneros, incluídas em 46 famílias (Tabela 2).

Tabela 2. Famílias e espécies amostradas nas cinco áreas de cerrado sensu stricto, ordenadas alfabeticamente por família

\begin{tabular}{|c|c|}
\hline Famílias/ Espécies & Famílias/ Espécies \\
\hline 1. Anacardiaceae & 4. $\quad$ Arecaceae \\
\hline Anacardium humile A.St.-Hil & Acrocomia aculeata (Jacq.) Lodd. ex \\
\hline Anacardium occidentale $\mathrm{L}$. & Mart. \\
\hline Astronium fraxinifolium Schott & Attalea maripa (Aubl.) Mart. \\
\hline Myracrodruon urundeuva Allemão & Oenocarpus distichus Mart. \\
\hline Schinus sp. & 5. Asteraceae \\
\hline Thyrsodium spruceanum Benth. & Moquiniastrum polymorphum (Less.) \\
\hline 2. Annonaceae & G. Sancho \\
\hline Annona coriacea Mart. & Piptocarpha rotundifolia (Less.) Baker \\
\hline Annona sp. & 6. Bignoniaceae \\
\hline Annona sylvatica A.St.-Hil. & Handroanthus albus (Cham.) Mattos \\
\hline Duguetia lanceolata A.St.-Hil. & Handroanthus chrysotrichus (Mart. \\
\hline Xylopia aromatica (Lam.) Mart. & ex DC.) Mattos \\
\hline 3. Apocynaceae & Handroanthus impetiginosus (Mart. \\
\hline Aspidosperma macrocarpon Mart. & ex DC.) Mattos \\
\hline Hancornia speciosa Gomes & Handroanthus ochraceus (Cham.) \\
\hline Himatanthus obovatus (Müll. Arg.) Woodson & Mattos \\
\hline
\end{tabular}

Famílias/ Espécies

Handroanthus serratifolius (Vahl)

S.Grose

Tabebuia aurea (Silva Manso) Benth. \& Hook.f. ex S.Moore

Tabebuia rosea (Bertol.) Bertero ex A.DC.

Tabebuia sp.

7. Boraginaceae

Cordia trichotoma (Vell.) Arráb. ex Steud.

8. Burseraceae

Protium heptaphyllum (Aubl.)

Marchand

Protium sp.

Trattinnickia rhoifolia Willd.

9. Calophyllaceae

Kielmeyera coriacea Mart. \& Zucc. 


\begin{tabular}{|c|c|c|}
\hline Famílias/ Espécies & Famílias/ Espécies & Famílias/ Espécies \\
\hline 10. Caricaceae & 20. Hypericaceae & 32. Myrtaceae \\
\hline Carica sp. & Vismia guianensis (Aubl.) Choisy & Eugenia dysenterica (Mart.) DC. \\
\hline 11. Caryocaraceae & 21. Icacinaceae & Marlierea racemosa (Vell.) Kiaersk. \\
\hline Caryocar brasiliense Cambess. & Emmotum nitens (Benth.) Miers & Myrcia fenzliana O.Berg \\
\hline 12. Chrysobalanaceae & 22. Lamiaceae & Myrcia splendens (Sw.) DC. \\
\hline Exellodendron cordatum (Hook.f.) Prance & Aegiphila sp. & Psidium cattleianum Sabine \\
\hline Hirtella ciliata Mart. \& Zucc. & Vitex megapotamica & Psidium guyanense Pers. \\
\hline 13. Clusiaceae & Moldenke & Psidium myrtoides O. Berg \\
\hline Platonia insignis Mart. & Vitex polygama Cham. & 33. Nyctaginaceae \\
\hline 14. Combretaceae & 23. Lauraceae & Guapira graciliflora \\
\hline Buchenavia tomentosa Eichler & Linharia tinctoria Arruda ex H.Kost. & Schmidt) Lundell \\
\hline Terminalia argentea Mart. & Mezilaurus itauba (Meisn.) Taub. ex & Guapira noxia (Netto) Lundell \\
\hline Terminalia glabrescens Mart. & $\mathrm{Mez}$ & 34. Ochnaceae \\
\hline Terminalia phaeocarpa Eichler & Nectandra sp. & Ouratea hexasperma (A.St.-Hil.) Baill. \\
\hline 15. Connaraceae & 24. Lecythidaceae & Ouratea sp. \\
\hline Connarus suberosus Planch. & Eschweilera ovata (Cambess.) Mart. & 35. Proteaceae \\
\hline 16. Dilleniaceae & ex Miers & Panopsis rubescens (Pohl) Rusby \\
\hline Curatella americana $\mathrm{L}$. & Cariniana rubra Gardner ex Miers & Roupala montana var. brasiliensis \\
\hline Davilla elliptica A.St.-Hil. & 25. Loganiaceae & (Klotzsch) K.S.Edwards \\
\hline 17. Ebenaceae & Strychnos pseudoquina A.St.-Hil. & 36. Rubiaceae \\
\hline Diospyros hispida A.DC. & 26. Lythraceae & Genipa americana $\mathrm{L}$. \\
\hline Diospyros ov & Lafoensia pacari A.St.-Hil. & Guettarda viburnoides Cham. \&Schltdl. \\
\hline 18. Euphor & Lafoensia sp. & Tocoyena formosa (Cham. \& Schltdl.) \\
\hline Mabea pohliana (Benth.) Müll.Arg. & Physocalymma scaberrimum Pohl & K.Schum. \\
\hline Maprounea guianensis Aubl. & 27. Malpighiaceae & 37. Rutaceae \\
\hline Sapium glandulosum (L.) Morong & Byrsonima basiloba A.Juss. & Zanthoxylum rhoifolium Lam. \\
\hline 19. Fabaceae & Byrsonima sericea DC. & 38. Salicaceae \\
\hline Albizia niopoides (Spruce ex Benth.) Burkart & Byrsonima sp. & Casearia sylvestris $\mathrm{Sw}$. \\
\hline Anadenanthera peregrina var. falcata & 28. Malvaceae & 39. Sapindaceae \\
\hline (B.) Altschul & Apeiba tibourbou Aubl. & Magonia pubescens A.St.-Hil. \\
\hline Andira anthelmia (Vell.) & Ceiba speciosa (A.St.-Hil.) Ravenna & Sapindus saponaria $\mathrm{L}$. \\
\hline (Vogel) J.F.Macbr. & Eriotheca gracilipes (K.Schum.) & 40. Sapotaceae \\
\hline Bowdichia virgilioides Kunth & A.Robyns & Pouteria ramiflora (Mart.) Radlk. \\
\hline Copaifera langsdorffii Desf. & Guazuma ulmifolia Lam. & Pouteria torta (Mart.) Radlk. \\
\hline lobium Benth. & Helicteres brevispira A.St.-Hil. & 41. Sima \\
\hline Dimorphandra mollis Benth. & Luehea divaricata Mart. \& Zucc. & Simarouba a \\
\hline Dipteryx alata Vogel & Pseudobombax grandiflorum (Cav.) & sicolor A.St.-Hil. \\
\hline siliquum (Vell.) Morong & A.Robyns & 42. $\mathrm{St}$. \\
\hline Erythrina & Pseudobombax longiflorum (Mart. \& & Styrax ferrugineus Nees \& Mart. \\
\hline aea stigonocarpa Mart. ex Hayne & Zucc.) A.Robyns & 43. Urtica \\
\hline Inga sp. & Theobroma speciosum Willd. ex & Cecropia hololeuca Miq. \\
\hline Leptolobiun & Spreng. & 44. Velloziaceae \\
\hline ium Vogel & 29. Melastomataceae & Vellozia sp. \\
\hline Machaerium & Mouriri elliptica Mart. & 45. Vochysiaceae \\
\hline Parkia platycephala Benth. & Mouriri pusa Gardner & Qualea grandiflora Mart. \\
\hline Peltogyne paniculata Benth. & Mouriri sp. & Qualea parviflora Mart. \\
\hline Peltophorum dubium (Spreng.) Taub. & 30. Meliaceae & Salvertia convallariodora A.St.-Hil. \\
\hline Plathymenia reticulata Benth. & Cabralea canjerana (Vell.) Mart. & Vochysia cinnamomea Pohl \\
\hline Pterodon emarginatus Vogel & Guarea guidonia (L.) Sleumer & Vochysia rufa Mart. \\
\hline Stryphnodendron adstringens (Mart.) Coville & 31. Moraceae & Vochysia sp. \\
\hline Tachigali aurea Tul. & Brosimum gaudichaudii Trécul & Vochysia tucanorum Mart. \\
\hline Tachigali vulgaris L.G.Sil & Clarisia racemosa Ruiz \& Pav. & 46. Winteraceae \\
\hline Vatairea macrocarpa (Benth.) Ducke & $\begin{array}{l}\text { Maclura tinctoria (L.) D.Don ex } \\
\text { Steud. }\end{array}$ & Drimys brasiliensis Miers \\
\hline
\end{tabular}

Na área 1 (A1) foram amostrados um total de 88 espécies, 40 famílias e 77 gêneros, na área 2 (A2) foram encontradas 53 espécies, 26 famílias e 51 gêneros, na área 3 (A3) foram inventariadas 45 espécies, 26 famílias e 43 gêneros, na área 4 (A4) foram catalogadas 62 espécies, 28 famílias e 58 gêneros e na área 5 (A5) a distribuição ocorreu com um total de 16 espécies, 09 famílias e 16 gêneros. Dentre todas as espécies amostradas no estudo apenas quinze foram identificadas em nível de gênero, sendo representadas por Aegiphila sp., Carica sp., Erythrina sp., Inga sp., Lafoensia sp., Mouriri sp., Nectandra sp., Tabebuia sp., Vellozia sp., Vochysia sp. (A1); Byrsonima sp. (A2); Ouratea sp. e Schinus sp. (A4); Anonna sp.e Protium sp. (A1 e A4). 
Em relação a quantidade de espécies amostradas, os valores encontrados para a A2 e A4, estão dentre o intervalo de 50 a 80 espécies, que geralmente é detectado no cerrado sensu stricto, no estado do Tocantins (Ferreira et al., 2017; Torres et al., 2017). Valores superiores a 80 espécies, como na área 1, foram encontrados em trabalhos como o de Aguiar et al. (2018) e Ferreira et al. (2017), na região Sul do Tocantins, demonstrando assim uma maior quantidade de espécies.

Os valores de riqueza observados para as áreas avaliadas corroboram com os encontrados em outras áreas de cerrado sensu stricto (Fina \& Monteiro, 2013; Nettesheim et al., 2010; Pedreira et al., 2011); entretanto, os valores de riqueza das áreas avaliadas corroboram com os valores das áreas de cerradão (Alves et al., 2013; Kunz et al., 2009). Dessa forma, pode-se inferir que o número de espécies encontradas nas áreas A1, A2 e A4 são altas, apresentando alta riqueza, o que possivelmente é um indicativo de bom estado de conservação das áreas avaliadas, uma vez que não foram observadas atividades antrópicas que pudessem interferir de forma negativa nos valores de riqueza, como o corte seletivo de certas espécies, ocorrência de fogo, atividades de cunho extrativista ou pastoreio, entretanto, a variação da riqueza das espécies pode ser explicado pelos diferentes tamanhos de parcelas.

Outro ponto que contribui para os valores de riqueza das áreas pode-se atribuir à localização geográfica do Estado do Tocantins, pois este Estado encontra-se em uma área de ecótono entre os Biomas Cerrado e Floresta Amazônica. Souza et al. (2008) e Ferreira et al. (2015) afirmam que, independentemente da sua origem florística, tanto o Cerrado quanto os outros biomas trocaram espécies ao longo do processo de adaptação da flora, sendo esse processo mais visível nas áreas de transição.

Quanto às famílias botânicas de um total de 40 famílias presentes na área (A1) 50,0\% foram representadas por uma única espécie, na área 2 (A2) 26 famílias, ou 65,4\%, na área 3 (A3) 26 famílias, ou 73,1\%, na área 4 (A4) 28 famílias com 57,1\% e na área 5 (A5) 9 famílias representando 77,8\%. Das 40 famílias encontradas na A1, Fabaceae apresentou maior riqueza (17 espécies), seguida por Malvaceae (6 espécies), Bignoniaceae (5 espécies), Annonaceae (4 espécies), Apocynaceae, Lauraceae e Myrtaceae (3 espécies). Já na A2, das 26 famílias amostradas, Fabaceae também apresentou maior riqueza (13 espécies), seguida por Vochysiaceae (4 espécies), Anacardiaceae, Annonaceae e Malvaceae (3 espécies). Na A3 foram identificadas 26 famílias, sendo que Fabaceae mais uma vez se destacou quanto à riqueza, apresentando o maior valor (11 espécies), seguida por Bignoniaceae, Malvaceae e Vochysiaceae (3 espécies). Dentre as 28 famílias identificadas na A4, as famílias mais representativas foram Fabaceae (14 espécies), Malvaceae (4 espécies), Myrtaceae (4 espécies), Anacardiaceae, Apocynaceae, Bignoniaceae e Vochysiaceae ( 3 espécies). E por fim na A5 onde foram identificadas 9 famílias onde Fabaceae também foi a mais representativa (6 espécies), seguida por Apocynaceae, Chrysobalanaceae, Lythraceae, Malpighiaceae, Malvaceae, Myrtaceae, Nyctaginaceae e Vochysiaceae com apenas uma espécie respectivamente.

O sucesso adaptativo das espécies da família Fabaceae pode estar relacionado ao estabelecimento de relações simbióticas com microrganismos, que aumentam a capacidade de absorção de fósforo e nitrogênio, elementos encontrados em baixas concentrações nos solos oligotróficos do Domínio Cerrado e que são fundamentais para o crescimento e estabelecimento das espécies (Franco, 2002; Silva Neto et al., 2016). Enquanto Vochysiaceae apresenta forte capacidade de acumulação de alumínio, por isso tende a ser encontrado um alto número de indivíduos desta família no Cerrado (Franco, 2002; Silva Neto et al., 2016).

A matriz para a análise de similaridade florística englobou 264 espécies registradas nas cinco áreas de cerrado sensu stricto, sendo área 1 (A1) 88 espécies, área 2 (A2) 53 espécies, área 3 (A3) 45 espécies, área 4 (A4) 62 espécies e área 5 (A5) 16 espécies. Dessa forma, após a análise de agrupamento formaram-se quatro grupos (A, B, C e D) para o índice de Sorensen (Figura 1).

O grupo (A), constituído pelas áreas 1 e 4, apresentaram maior similaridade em torno de 57,3\%, além de compartilhar 43 espécies de ligação Andira anthelmia (Vell.) Benth., Andira anthelmia (Vell.) Benth., Anonna sp., Aspidosperma macrocarpon Mart., Bowdichia virgilioides Kunth, Byrsonima basiloba A.Juss., Caryocar brasiliense Cambess., Clarisia racemosa Ruiz \& Pav., Copaifera langsdorffii Desf., Cordia trichotoma (Vell.) Arráb. ex Steud., Dalbergia miscolobium Benth., Dimorphandra mollis Benth., Diospyros hispida A.DC., Drimys brasiliensis Miers, Eriotheca gracilipes (K.Schum.) A.Robyns, Guarea guidonia (L.) Sleumer, Guazuma ulmifolia Lam., Hancornia speciosa Gomes, Handroanthus albus (Cham.) Mattos, Handroanthus serratifolius (Vahl) S.Grose, Himatanthus 
obovatus (Müll. Arg.) Woodson, Hymenaea stigonocarpa Mart. ex Hayne, Luehea divaricata Mart. \& Zucc., Mabea pohliana (Benth.) Müll.Arg., Maclura tinctoria (L.) D.Don ex Steud., Magonia pubescens A.St.-Hil., Mouriri elliptica Mart., Myrcia fenzliana O.Berg, Panopsis rubescens (Pohl) Rusby, Physocalymma scaberrimum Pohl, Plathymenia reticulata Benth., Protium sp., Psidium myrtoides O.Berg, Pterodon emarginatus Vogel, Qualea grandiflora Mart., Qualea parviflora Mart., Simarouba amara Aubl., Styrax ferrugineus Nees \& Mart., Tabebuia aurea (Silva Manso) Benth. \& Hook.f. ex S.Moore, Tachigali aurea Tul., Tachigali vulgaris L.G.Silva \& H.C.Lima, Vatairea macrocarpa (Benth.) Ducke, Vochysia tucanorum Mart. e Xylopia aromatica (Lam.) Mart.

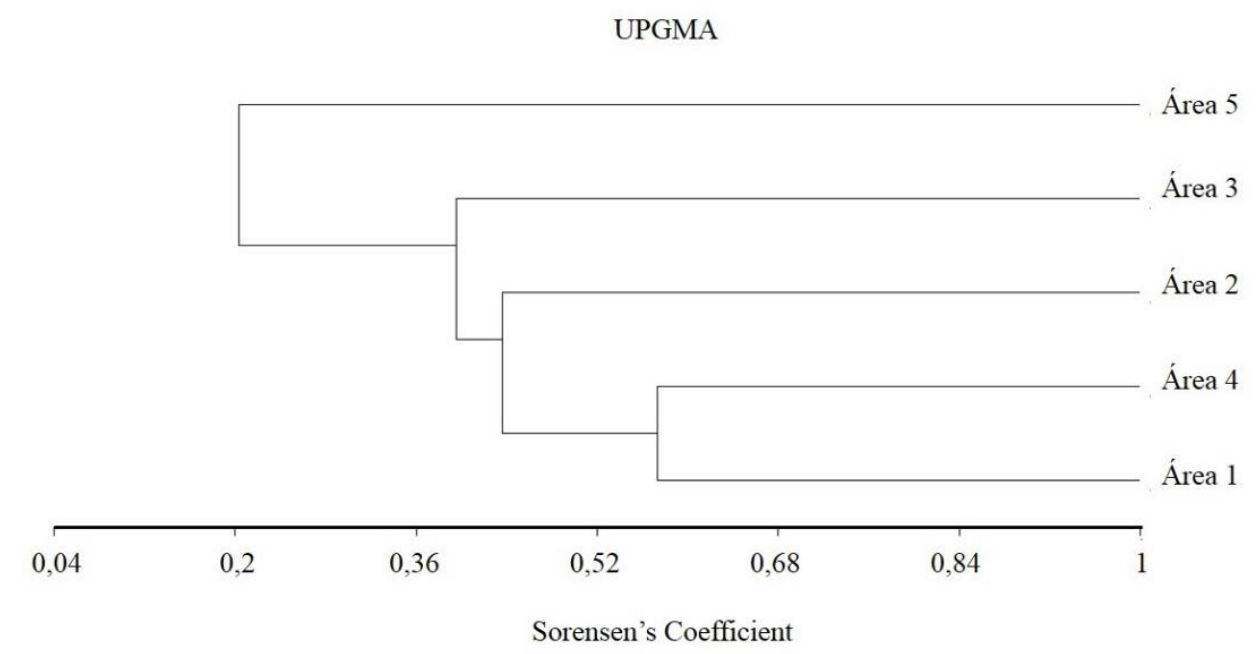

Figura 1. Dendrograma da análise de agrupamentos por médias não-ponderadas (UPGMA) das similaridades florísticas (Sørensen) entre 5 áreas de cerrado sensu stricto do Estado de Tocantins.

O grupo (B), formado pela área 2 apresentou uma similaridade entre 0,4 e 0,52, além de compartilhar 21 espécies de ligação Andira anthelmia (Vell.) Benth., Aspidosperma macrocarpon Mart., Bowdichia virgilioides Kunth, Caryocar brasiliense Cambess., Dimorphandra mollis Benth., Diospyros hispida A.DC., Eriotheca gracilipes (K.Schum.) A.Robyns, Guazuma ulmifolia Lam., Hancornia speciosa Gomes, Himatanthus obovatus (Müll. Arg.) Woodson, Hymenaea stigonocarpa Mart. ex Hayne, Magonia pubescens A.St.-Hil., Plathymenia reticulata Benth., Pterodon emarginatus Vogel, Qualea grandiflora Mart., Qualea parviflora Mart., Tabebuia aurea (Silva Manso) Benth. \& Hook.f. ex S.Moore, Tachigali aureaTul.,Tachigali vulgaris L.G.Silva \& H.C.Lima, Vatairea macrocarpa (Benth.) Ducke e Xylopia aromatica (Lam.) Mart. Pode-se inferir que a similaridade florística do grupo B esteja relacionada com fatores ambientais semelhantes (altitude, tipo de solo e clima) (SEPLAN, 2015).

O grupo (C), constituído pela área 3 e grupo $B$, apresentaram similaridade entre 0,36 e 0,42 , além de compartilhar 13 espécies de ligação as espécies Andira anthelmia (Vell.) Benth., Bowdichia virgilioides Kunth, Caryocar brasiliense Cambess., Guazuma ulmifolia Lam., Hancornia speciosa Gomes, Hymenaea stigonocarpa Mart. ex Hayne, Magonia pubescens A.St.-Hil., Plathymenia reticulata Benth., Qualea grandiflora Mart., Tabebuia aurea (Silva Manso) Benth. \& Hook.f. ex S.Moore, Tachigali vulgaris L.G.Silva \& H.C.Lima, Vatairea macrocarpa (Benth.) Ducke e Xylopia aromatica (Lam.) Mart.

Segundo Kent \& Coker (1992) similaridade acima de 50\% são consideradas altas, dessa forma, podese afirmar que a similaridade entre as parcelas amostradas nas áreas (01 e 04) e (02 e 04) foram elevadas. As áreas 01 e 02, apesar de possuírem as mesmas condições climáticas, apresentaram menor número de espécies de ligação entre si quando comparadas ao Grupo A, indicando que além da altitude e clima semelhante as características edáficas também foram importantes para determinar a vegetação que ocorre num determinado local.

O Grupo C apresentou baixa similaridade florística entre 0,36 e 0,42, compartilhando 13 espécies de ligação. Esta pouca semelhança vegetacional da área 03 em relação às áreas 01,02 e 04 pode ser devido as características edáficas, como por exemplo atributos como a fertilidade, topografia, teor de matéria orgânica, drenagem, salinidade, acidez e textura do solo. 
O grupo $\mathrm{D}$, formado pela área 5 é o menos similar, todavia, o mesmo faz ligação com as demais áreas, tal diferença florística pode ser explicada pelas características edáficas associada a altitude e precipitação média anual local.

A heterogeneidade observada entre as áreas avaliadas indica que as espécies desta fisionomia de cerrado sensu stricto se caracterizam pela distribuição espacial em "mosaicos", com um limite máximo de 100 espécies por área estudada, e mesmo as populações existentes em áreas próximas apresentam-se floristicamente e estruturalmente diferenciadas (Lopes et al., 2010).

\section{Conclusões}

A composição florística das áreas estudadas em diferentes regiões no estado do Tocantins é coerente com as demais formações savânicas predominantes no cerrado sensu stricto da região Norte do Brasil, Amazônia Legal.

As áreas de cerrado sensu stricto analisadas apresentaram alta heterogenia de espécies, onde a família Fabaceae apresentou maior riqueza em todas as áreas estudadas.

Após analisar as informações ambientais das cinco áreas e cruzá-las com os resultados alcançados, foi possível perceber que as características edáficas, condições climáticas e altitude são fatores importantes na similaridade florística da fisionomia de cerrado sensu stricto.

\section{Referências}

Aguiar, B. A., Camargo, M. O., Ferreira, R. Q. S., Teixeira, Paulo Ricardo Silva, R. R., \& Souza, P. B. (2018). Florística e estrutura do componente arbustivo-arbóreo de um remanescente de cerrado sensu stricto, Gurupi, Tocantins. Revista Verde de Agroecologia e Desenvolvimento Sustentável, 13(1), 4551. DOI: https://doi.org/10.18378/rvads.v13i1.5271

Alves, H. R., Prado Júnior, J., Lopes, S., Silva, P. P., Peppe, F. B., \& Schiavini, I. (2013). Fitossociologia e grupos ecológicos da comunidade lenhosa em um remanescente de cerradão em Uberlândia, MG. Caminhos de Geografia, 14(46), 236-245.

APG. Angiosperm Phylogeny Group. (2009). An update of the Angiosperm Phylogeny Group classification for the orders and families of flowering plants: APG III. Botanical Journal of the Linnean Society, 161, 105-121. DOI: https://doi.org/10.1111/j.1095-8339.2009.00996.x

BRASIL. Ministério do Meio Ambiente. (2019). O bioma Cerrado. Disponível em: <http://www.mma.gov.br/biomas /cerrado>. Acesso em 10 jan. 2019.

Ferreira, R. Q. S., Camargo, M. O., Souza, P. B., \& Andrade, V. C. L. (2015). Fitossociologia e estrutura diamétrica de um cerrado sensu stricto, Gurupi-TO. Revista Verde de Agroecologia e Desenvolvimento Sustentável, 10(1), 229-235. DOI: https://doi.org/10.20873/uft.2359$\underline{3652.2016 \mathrm{v} 3 \mathrm{nespp} 22}$

Ferreira, R. Q. S., Camargo, M. O., Teixeira, P. R., Souza, P. B., \& Souza, D. J. (2017). Diversidade florística do estrato arbustivo arbóreo de três áreas de cerrado sensu stricto, Tocantins. DESAFIOSRevista Interdisciplinar Da Universidade Federal Do Tocantins, 4(2), 69-82. DOI: https://doi.org/10.20873/uft.2359-3652.2017v4n2p69

Fina, B. G., \& Monteiro, R. (2013). Análise da estrutura arbustivo-arbórea de uma área de cerrado sensu stricto, município de Aquidauana-Mato Grosso do Sul. Revista Árvore, 37(4), 577-585. DOI: https://doi.org/10.1590/S0100-67622013000400001

Franco, A. C. (2002). Ecophysiology of woody plants. In P. S. Oliveira \& R. J. Marquis (Eds.), The cerrado of Brazil: ecology and natural history of Neotropical savanna. Columbia University Press.

Kent, M., \& Coker, P. (1992). Vegetation Description and Analysis: A Practical Approach Belhaven Press. Belhaven.

Kovach, W. L. (2007). Kovach computing services. MVSP Plus Version, 3, 137.

Kunz, S. H., Ivanauskas, N. M., \& Martins, S. V. (2009). Estrutura fitossociológica de uma área de cerradão em Canarana, Estado do Mato Grosso, Brasil. Acta Scientiarum. Biological Sciences, 31(3), 255-261. DOI: https://doi.org/10.4025/actascibiolsci.v31i3.1625 
Lopes, J. F. B., Andrade, E. M., Lobato, F. A. O., Palácio, H. A. Q., \& Arraes, F. D. D. (2010). Deposição e decomposição de serapilheira em área da Caatinga. Revista Agro@Ambiente, 3(2), 72-79. DOI: https://doi.org/10.18227/1982-8470ragro.v3i2.252

Magurran, A. E. (1998). Population differentiation without speciation. Philosophical Transactions of the Royal Society of London. Series B: Biological Sciences, 353(1366), 275-286. DOI: https://doi.org/10.1098/rstb.1998.0209

Mueller-Dombois, D., \& Ellenberg, H. (1974). Aims and methods of vegetation ecology. John Wiley \& Sons. DOI: https://doi.org/10.2307/213332

Nettesheim, F. C., Carvalho, D. C., Fonseca, C. C., Nunes, R. S., Cavalcanti, D. M., Gabriel, M. M., \& Menezes, L. F. T. (2010). Estrutura e florística do estrato arbóreo no cerrado sensu stricto de Buritis, Minas Gerais, Brasil. Rodriguésia, 61(4), 731-748. DOI: https://doi.org/10.1590/2175$\underline{7860201061413}$

Pedreira, F. R. B., Alves, L. R., Lolis, S. F., \& Viana, R. H. O. (2011). Composição florística e fitossociologia de espécies arbóreas em uma área de cerrado stricto sensu no Município de Porto Nacional, TO. Global Science and Technology, 4(1), 8-15. DOI: https://doi.org/10.14688/1984$\underline{3801 / g s t . v 4 n 1 p 8-15}$

Purvis, A., \& Hector, A. (2000). Getting the measure of biodiversity. Nature, 405(6783), 212-219. DOI: https://doi.org/10.1038/35012221

Ratter, J. A., Bridgewater, S., \& Ribeiro, J. F. (2003). Analysis of the floristic composition of the Brazilian cerrado vegetation III: comparison of the woody vegetation of 376 areas. Edinburgh Journal of Botany, 60(1), 57-109. DOI: https://doi.org/10.1017/s0960428603000064

REFLORA - Plantas do Brasil: Herbário Virtual do Brasil. (2020). Jardim Botânico do Rio de Janeiro. Disponível em: 〈http://floradobrasil.jbrj.gov.br/reflora/floradobrasil/FB115>. Acesso em: 20 ago. 2018.

Sano, E. E., Rosa, R., Brito, J. L. S., \& Ferreira, L. G. (2010). Land cover mapping of the tropical savanna region in Brazil. Environmental Monitoring and Assessment, 166(1-4), 113-124. DOI: https://doi.org/10.1007/s10661-009-0988-4

SEPLAN - Secretaria do Planejamento e Orçamento. (2015). Perfil socioeconômico. TOCANTINS (Governo do Estado do Tocantins). Disponível em: <http://seplan.to.gov.br/estatistica/perfilsocioeconomico/versao-2015/>. Acesso em: 17 ago. 2018.

Shepherd, G. J. (2010). FITOPAC. Versão 2.1. Campinas, SP: Departamento de Botânica, Universidade Estadual de Campinas-UNICAMP. Universidade Estadual de Campinas.

Silva Neto, V. L., Oliveira, A. L., Ferreira, R. Q. S., Souza, P. B., \& Viola, M. R. (2016). Fitossociologia e distribuição diamétrica de uma área de Cerrado sensu stricto, Dueré-TO. Revista de Ciências Ambientais, 10(1), 91-106. DOI: https://doi.org/10.18316/1981-8858.16.24

Sneath, P. H., \& Sokal, R. R. (1966). Numerical taxonomy. WH Freeman and Co, 27, 75-85. DOI: https://doi.org/10.2307/1219070

Souza, P. B., Alves, J. A., Silva, A. F., \& Souza, A. L. (2008). Composição florística da vegetação arbórea de um remanescente de cerração, Paraopeba, MG. Revista Árvore, 32(4), 781-790. DOI: https://doi.org/10.1590/s0100-67622008000400020

Torres, D. M., Fontes, M. A. L., \& Samsonas, H. do P. (2017). Soil-vegetation relationships in structuring cerrado sensu stricto communities in southern Minas Gerais, Brazil. Rodriguésia, 68(1), 115-128. DOI: https://doi.org/10.1590/2175-7860201768121

Histórico do artigo:

Recebido: 28 de setembro de 2020 Aprovado: 23 de outubro de 2020.

Disponível online: 12 de janeiro de 2021.
Licenciamento: Este artigo é publicado na modalidade Acesso Aberto sob a licença Creative Commons Atribuição 4.0 (CC-BY 4.0), a qual permite uso irrestrito, distribuição, reprodução em qualquer meio, desde que o autor e a fonte sejam devidamente creditados. 